\title{
Momentum transfer to small particles by passing electron beams
}

\author{
F. J. García de Abajo \\ Centro Mixto CSIC-UPV/EHU and Donostia International Physics Center (DIPC), Aptdo. 1072, 20080 San Sebastián, Spain
}

(Received 29 January 2004; published 29 September 2004)

\begin{abstract}
The force exerted on nanoparticles and atomic clusters by fast passing electrons like those employed in transmission electron microscopes are calculated and integrated over time to yield the momentum transferred from the electrons to the particles. Numerical results are offered for metallic and dielectric particles of different sizes ( $0-500 \mathrm{~nm}$ in diameter) as well as for carbon nanoclusters. Results for both linear and angular momentum transfers are presented. For the electron beam currents commonly employed in electron microscopes, the time-averaged forces are shown to be comparable in magnitude to laser-induced forces in optical tweezers. This opens up the possibility to study optically trapped particles inside transmission electron microscopes.
\end{abstract}

DOI: 10.1103/PhysRevB.70.115422

PACS number(s): 73.20.Mf, 33.80.Ps, 42.50.Vk, 78.67.Bf

\section{INTRODUCTION}

Electromagnetic forces in optical tweezers are currently employed to trap small particles ranging in size from nanometers to several microns, ${ }^{1,2}$ and to manipulate them in all spatial directions. ${ }^{3,4}$ This type of force is also used to characterize the elastic properties of deformable tiny objects (e.g., living cells ${ }^{5}$ ), to obtain quantitative information on mechanical properties at small length scales, ${ }^{2}$ and in general, to fix the position of those particles so that they can be manipulated at will.

In this context, transmission electron microscopy offers a potentially useful tool to study optically trapped particles, providing excellent spatial resolution (sometimes below $1 \AA$ ) when subnanometer electron beams are employed, ${ }^{6}$ while allowing spectroscopic characterization with sub-electronvolt accuracy. Actually, transmission electron microscopes are routinely exploited to probe local optical response properties, ${ }^{7}$ and more recently, also to determine photonic structures of complex materials. ${ }^{8}$

A major problem that may arise when combining electron microscopy with optical tweezers or other types of optical trapping (e.g., optical lattices ${ }^{9-11}$ ) is that the passing electrons can kick the particles out of the trapping locations (see Fig. 1). In this work, we show that the momentum transferred from the passing electrons to the particles can be well below the threshold needed to kick them out for commonly employed trapping laser intensities, although a detailed comparison between trapping forces and electron-induced forces suggests that both weak and strong perturbation regimes are possible depending on the distance between the particles and the beam, all of them within the range that allows a sufficiently large electron-particle interaction as to perform electron energy loss spectroscopy (EELS) with significant statistics for in vасио optically trapped particles.

The moving electrons can be in fact regarded as a source of evanescent electromagnetic field that probes the sample locally, and in this sense, they can be also used to produce deformation in elastic particles, oscillations of trapped particles around their equilibrium positions, and other interesting effects associated to the transfer of momentum within accurately controlled spatial regions.

The present work addresses, in a quantitative way, the issue of both linear and angular momentum transfer from an electron beam to small particles. This applies to optically trapped particles, as mentioned above, but also to other forms of trapping, like particles deposited on solid substrates, or particles trapped by a tip [e.g., in a scanning tunnel microscope (STM) set up].

\section{THEORY}

The electromagnetic force exerted on a particle in vacuum is given by the integral of Maxwell's stress tensor over a surface $S$ embedding the particle ${ }^{12}$ as

$$
\begin{aligned}
\mathbf{F}(t)= & \frac{1}{4 \pi} \int_{S} d \mathbf{s}[\mathbf{E}(\mathbf{s}, t) \mathbf{E}(\mathbf{s}, t) \cdot \hat{\mathbf{n}}+\mathbf{H}(\mathbf{s}, t) \mathbf{H}(\mathbf{s}, t) \cdot \hat{\mathbf{n}} \\
& \left.-\frac{\hat{\mathbf{n}}}{2}\left(|\mathbf{E}(\mathbf{s}, t)|^{2}+|\mathbf{H}(\mathbf{s}, t)|^{2}\right)\right],
\end{aligned}
$$

where $\hat{\mathbf{n}}$ is the surface normal and Gaussian units are used. The momentum transferred to the particle, $\Delta \mathbf{p}$, is obtained by integrating of $\mathbf{F}(t)$ over the time. This yields

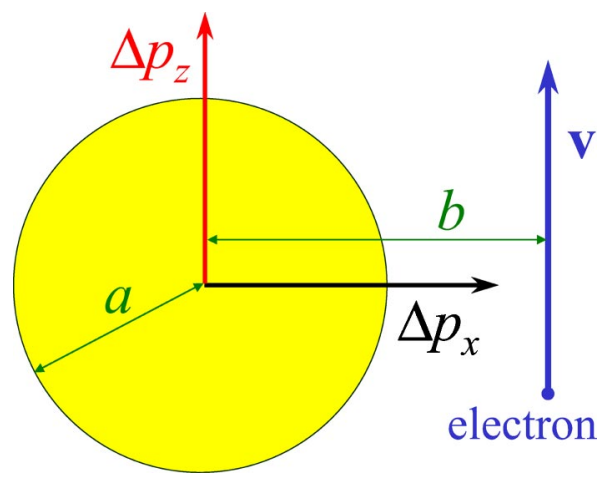

FIG. 1. (Color online). Schematic representation of the process considered in this work: a fast electron moving with impact parameter $b$ and velocity $v$ with respect to a polarizable particle transfers momentum $\Delta \mathbf{p}=\left(\Delta p_{x}, \Delta p_{z}\right)$ to the particle via electromagnetic interaction. 


$$
\Delta \mathbf{p}=\int \mathbf{F}(t) d t=\int_{0}^{\infty} \mathbf{F}(\omega) d \omega
$$

where

$$
\begin{aligned}
\mathbf{F}(\omega)= & \frac{1}{4 \pi^{2}} \operatorname{Re}\left\{\int_{S} d \mathbf{s}[\mathbf{E}(\mathbf{s}, \omega)[\mathbf{E}(\mathbf{s}, \omega) \cdot \hat{\mathbf{n}}] *+\mathbf{H}(\mathbf{s}, \omega)\right. \\
& \left.\left.\times[\mathbf{H}(\mathbf{s}, \omega) * \cdot \hat{\mathbf{n}}) *-\frac{\hat{\mathbf{n}}}{2}\left(|\mathbf{E}(\mathbf{s}, \omega)|^{2}+|\mathbf{H}(\mathbf{s}, \omega)|^{2}\right]\right]\right\},
\end{aligned}
$$

and the Fourier transform is defined as $\mathbf{E}(\mathbf{r}, \omega)$ $=\int d t \mathbf{E}(\mathbf{r}, t) \exp \{i \omega t\}$.

The force acting on the particle is due in part to radiation emitted as a result of interaction with the electron and in part to the reaction force experienced by the projectile. For small particles, the effect of radiation emission is negligible and the trajectory is deflected by an angle $\approx \Delta p / m v$, where $m$ and $v$ are the mass and velocity of the electron. Nonretarded calculations have shown that this angle is too small to be easily measured. ${ }^{13}$

\section{A. Small particles}

Let us first consider a small isotropic particle sufficiently far away from the electron beam as to neglect higher multipoles beyond induced dipoles. The particle is then characterized by its frequency-dependent polarizability $\alpha(\omega)$, and the force exerted by each frequency component of the external field $\mathbf{E}(\mathbf{r}, \omega)$ reduces to ${ }^{14}$

$$
\mathbf{F}(\omega)=\operatorname{Re}\left\{\alpha \sum_{j} E_{j}^{\operatorname{ext}}(\mathbf{r}, \omega) \nabla\left[E_{j}^{\text {ext }}(\mathbf{r}, \omega)\right]^{*}\right\} .
$$

This expression can be derived from Eq. (2) by considering an integration surface arbitrarily close to the object and by using the expressions for the electric and magnetic fields induced by a small polarizable particle in terms of its polarizability $\alpha$. For an electron moving with velocity $v$ toward a positive $z$ direction and passing by the origin at $t=0$, the external field is readily calculated from Maxwell's equations to yield

$$
\mathbf{E}^{\operatorname{ext}}(\mathbf{r}, \omega)=\frac{-2 e \omega}{v^{2} \gamma} \mathrm{e}^{\mathrm{i} \omega z z l v}\left[K_{1}\left(\frac{\omega R}{v \gamma}\right) \frac{\mathbf{R}}{R}-\frac{\mathrm{i}}{\gamma} K_{0}\left(\frac{\omega R}{v \gamma}\right) \hat{\mathbf{z}}\right],
$$

where $\mathbf{R}=(x, y)$ and $\gamma=1 / \sqrt{1-v^{2} / c^{2}}$. Inserting Eq. (4) into Eq. (3), one obtains

$$
\mathbf{F}(\omega)=\frac{2 e^{2} \omega^{3}}{v^{5} \gamma^{3}}\left[-\operatorname{Re}\{\alpha\} f^{\prime}\left(\frac{\omega b}{v \gamma}\right) \hat{\mathbf{x}}+2 \gamma \operatorname{Im}\{\alpha\} f\left(\frac{\omega b}{v \gamma}\right) \hat{\mathbf{z}}\right],
$$

where

$$
f(\zeta)=K_{1}^{2}(\zeta)+K_{0}^{2}(\zeta) / \gamma^{2},
$$

and the particle is taken to be situated at $\mathbf{R}=(-b, 0)$ with respect to the beam (see Fig. 1).
Symmetry considerations lead to the conclusion that Rayleigh scattering of the external-electron evanescent field (4) produces a radiation pattern with inversion symmetry with respect to a plane perpendicular to the trajectory. This means that the overall transfer of momentum to the induced radiation is zero in the small-particle limit, so that $\Delta p_{z}$ accounts for all momentum transfer to the moving electron along $z$. Then, the contribution of each $\omega$ component to the electron energy loss rate is, within the nonrecoil approximation valid for sufficiently energetic electrons, $v F_{z}(\omega)$. Actually, one finds that the identity $v F_{z}(\omega)=\hbar \omega P(\omega)$ is satisfied, where $P(\omega)$ is the frequency-resolved loss probability as previously obtained for small particles. ${ }^{15}$ As a consequence, $F_{z}$ vanishes in the $\omega \rightarrow 0$ limit, since $P(\omega)$ remains finite.

This behavior is quite different from $F_{x}$, which goes to a finite value for small $\omega$ 's, namely, $F_{x}(\omega=0)$ $=4 e^{2} \operatorname{Re}\{\alpha(0)\} / v^{2} b^{3}$. (Incidentally, momentum transfer along $x$ produces negligible energy transfer in the nonrecoil approximation.) This latter formula can be used to derive a close expression for $\Delta p_{x}$ valid for arbitrarily large, finite objects in the large impact parameter limit. In that case, only small $\omega$ 's contribute to $\mathbf{F}(\omega)$, due to the effective exponential cut-off imposed by the modified Bessel functions $K_{0}$ and $K_{1}$. This means that only long wavelengths are relevant (to which the object appears as small), so that it can be described by its static polarizability. Then, the $\omega$ integral can be performed numerically to yield

$$
\Delta p_{x}=\left(5.55165 \gamma+\frac{1.85055}{\gamma}\right) \frac{e^{2} \operatorname{Re}\{\alpha(0)\}}{v b^{4}} .
$$

For comparison, the momentum transferred to a charge $e$ at a distance $b$ from the beam is $\Delta \mathbf{p}=-\left(2 e^{2} / b v\right) \hat{\mathbf{x}}$.

The large- $b$ limit given by Eq. (6) is compared in Fig. 2 with more detailed calculations that include higher-multipole moments, as described below. Also, the small particle limit of Eq. (5) is discussed in Fig. 3.

\section{B. Arbitrary size}

For larger particles or for close electron-particle encounters, higher multipoles become relevant in the induced forces. ${ }^{16}$ Then, it is convenient to express the evanescent field of the electron in terms of multipoles centered at the particle, so that the external electric and magnetic fields admit the following decomposition: ${ }^{15,17}$

$$
\mathbf{E}^{\mathrm{ext}}(\mathbf{r}, \omega)=\sum_{L}\left[\psi_{L}^{M, \mathrm{ext}} \mathbf{L}-\frac{\mathrm{i}}{k} \psi_{L}^{E, \text { ext }} \nabla \times \mathbf{L}\right] j_{L}(k \mathbf{r})
$$

and

$$
\mathbf{H}^{\mathrm{ext}}(\mathbf{r}, \omega)=-\sum_{L}\left[\psi_{L}^{E, \text { ext }} \mathbf{L}+\frac{\mathrm{i}}{k} \psi_{L}^{M, \text { ext }} \nabla \times \mathbf{L}\right] j_{L}(k \mathbf{r}),
$$

where $L=(l, m), k=\omega / c, j_{L}(k \mathbf{r})=\mathrm{i}^{l} j_{l}(k r) Y_{L}(\hat{\mathbf{r}}), \mathbf{L}=-\mathrm{i} \hbar \mathbf{r} \times \nabla$ is the orbital angular momentum operator, and $\psi_{L}^{\nu, \text { ext }}$ (for $\nu$ $=E, M)$ are multipole coefficients given by ${ }^{15,17}$ 


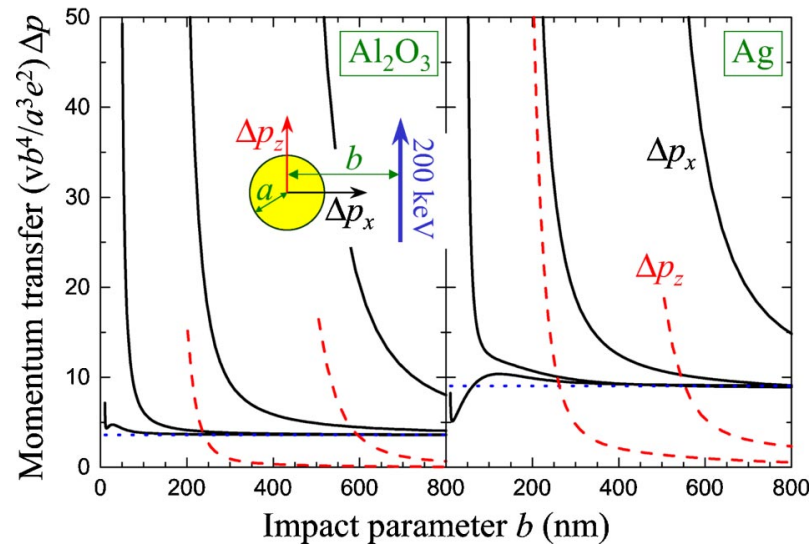

FIG. 2. (Color online). Momentum transfer to small spherical particles by a passing $200-\mathrm{keV}$ electron as a function of the distance from the trajectory to the center of the spheres $b$. The momentum transfer has been scaled using the velocity $v=0.7 c$, the sphere radius $a$, and the impact parameter $b$. The perpendicular component of the momentum transfer with respect to the trajectory $\Delta p_{x}$ (solid curves) has been represented for spheres of radius $a=10,50,200$, and $500 \mathrm{~nm}$ (notice the rapid increase in $\Delta p_{x}$ near $b=a$ ). The parallel component $\Delta p_{z}$ (dashed curves) is only shown for $a=200$ and $500 \mathrm{~nm}$. Dielectric alumina spheres and metallic silver spheres are considered (left and right plot, respectively), respectively. The large $b$ limit for perpendicular momentum transfer [Eq. (6)] is shown by horizontal dotted lines.

$$
\left[\begin{array}{c}
\psi_{L}^{M, \text { ext }} \\
\psi_{L}^{E \text {,ext }}
\end{array}\right]=\frac{-2 \pi \mathrm{i}^{1-l} e k}{l(l+1) \hbar c}\left[\begin{array}{c}
2 m A_{L} v / c \\
B_{L} / \gamma
\end{array}\right] K_{m}\left[\frac{\omega b}{v \gamma}\right],
$$

with

$$
\begin{gathered}
A_{L}=\sqrt{\frac{2 l+1}{\pi} \frac{(l-|m|) !}{(l+|m|) !}}(2|m|-1) ! ! \\
\times \frac{\mathrm{i}^{l+|m|} s_{m}}{(v / c)(v \gamma / c)^{|m|}} C_{l-|m|}^{(|m|+1 / 2)}\left(\frac{c}{v}\right), \\
B_{L}=A_{l, m+1} C_{+}-A_{l, m-1} C_{-},
\end{gathered}
$$

and

$$
C_{ \pm}=\sqrt{(l \pm m+1)(l \mp m)} .
$$

Here, $s_{m}=1$ if $m \geqslant 0, s_{m}=(-1)^{m}$ if $m<0$, and $C_{m}^{(v)}$ is the Gegenbauer polynomial. ${ }^{18}$ The impact parameter $b$ is defined in Fig. 1.

The induced field around the particle is given by similar expressions obtained by substituting $\psi_{L}^{\nu, \text { ext }}$ by coefficients $\psi_{L}^{\nu, \text { ind }}$, and $j_{l}$ by the Hankel function $h_{l}^{(+)} \cdot{ }^{19}$ In particular, $L$ $=(l, m)$ is conserved for spherical particles and one has a linear dependence $\psi_{L}^{\nu \text {,ind }}=t_{l}^{\nu} \psi_{L}^{\nu, \text { ext }}$, where $t_{l}^{\nu}$ are scattering matrices that are given by analytical expressions in the case of homogeneous particles of dielectric function $\epsilon$ and radius $a^{15}$

$$
t_{l}^{M}=\frac{-j_{l}\left(\rho_{0}\right) \rho_{1} j_{l}^{\prime}\left(\rho_{1}\right)+\rho_{0} j_{l}^{\prime}\left(\rho_{0}\right) j_{l}\left(\rho_{1}\right)}{h_{l}^{(+)}\left(\rho_{0}\right) \rho_{1} j_{l}^{\prime}\left(\rho_{1}\right)-\rho_{0}\left[h_{l}^{(+)}\left(\rho_{0}\right)\right]^{\prime} j_{l}\left(\rho_{1}\right)}
$$

and

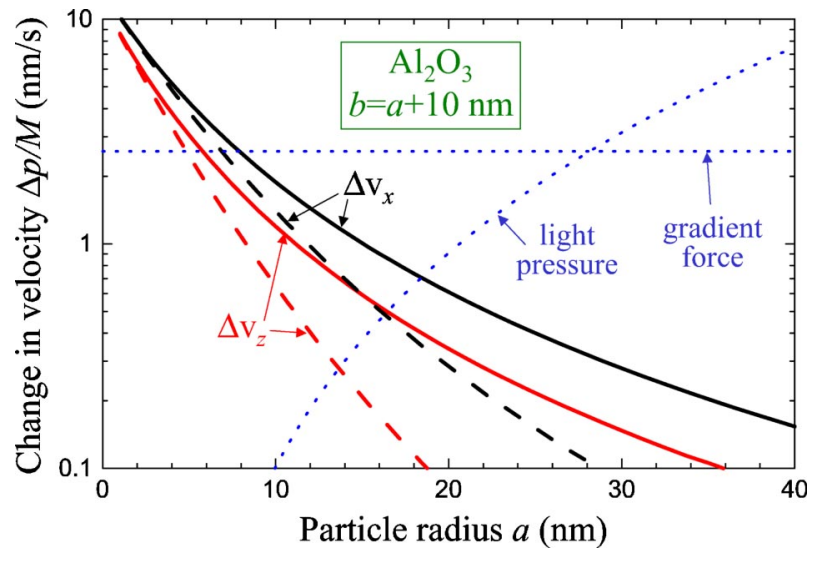

FIG. 3. (Color online). Particle size dependence of the momentum transfer normalized to the particle mass $M$ under the same conditions as in Fig. 2: small particle limit (dashed curves) versus full multipole calculation (solid curves). The particle is made of $\mathrm{Al}_{2} \mathrm{O}_{3}$ (density $\rho=4.02 \mathrm{~g} / \mathrm{cm}^{3}$ ), the electron energy is $200 \mathrm{keV}$, and the distance from the trajectory to the particle surface is $10 \mathrm{~nm}$. Dotted curves show the momentum transferred from light in an optical trap (see text for details).

$$
t_{l}^{E}=\frac{-j_{l}\left(\rho_{0}\right)\left[\rho_{1} j_{l}\left(\rho_{1}\right)\right]^{\prime}+\epsilon\left[\rho_{0} j_{l}\left(\rho_{0}\right)\right]^{\prime} j_{l}\left(\rho_{1}\right)}{h_{l}^{(+)}\left(\rho_{0}\right)\left[\rho_{1} j_{l}\left(\rho_{1}\right)\right]^{\prime}-\epsilon\left[\rho_{0} h_{l}^{(+)}\left(\rho_{0}\right)\right]^{\prime} j_{l}\left(\rho_{1}\right)},
$$

where $\rho_{0}=k a, \rho_{1}=\rho_{0} \sqrt{\epsilon}$ with $\operatorname{Im}\left\{\rho_{1}\right\}>0$, and the prime denotes differentiation with respect to $\rho_{0}$ and $\rho_{1}$.

At this point, it is convenient to write the operators $\mathbf{L}$ and $(1 / k) \nabla$ in matrix form. One finds

$$
\mathbf{L} j_{L}=\sum_{L^{\prime}} \mathbf{L}_{L L^{\prime}} j_{L^{\prime}}
$$

and

$$
\frac{1}{k} \nabla j_{L}=\sum_{L^{\prime}} \mathbf{N}_{L L^{\prime} j_{L^{\prime}}}
$$

respectively, where

$$
\begin{aligned}
\mathbf{L}_{L L^{\prime}}= & \hbar \delta_{l, l^{\prime}}\left[C_{+} \delta_{m+1, m^{\prime}}(\hat{\mathbf{x}}-\mathrm{i} \hat{\mathbf{y}}) / 2+C_{-} \delta_{m-1, m^{\prime}}(\hat{\mathbf{x}}+\mathrm{i} \hat{\mathbf{y}}) / 2\right. \\
& \left.+m \delta_{m, m^{\prime}} \hat{\mathbf{z}}\right],
\end{aligned}
$$

$$
\hat{\mathbf{z}} \cdot \mathbf{N}_{L L^{\prime}}=\mathrm{i} \delta_{m, m^{\prime}}\left(\delta_{l+1, l^{\prime}}+\delta_{l-1, l^{\prime}}\right) \frac{\left(l^{\prime}+m\right)\left(l^{\prime}-m\right)}{\left(2 l^{\prime}-1\right)\left(2 l^{\prime}+1\right)},
$$

and the $\hat{\mathbf{x}}$ and $\hat{\mathbf{y}}$ components of $\mathbf{N}$ are obtained from Eq. (8) by rotating the reference frame using rotation matrices for spherical harmonics. ${ }^{19}$ Exactly the same matrices as above apply to $\mathbf{L}$ and $(1 / k) \nabla$ acting on Hankel functions $h_{L}^{(+)}$. Furthermore, these matrices satisfy the properties $\mathbf{L}^{+}=\mathbf{L}$ and $\mathbf{N}^{+}=-\mathbf{N}$.

Now, the electric field admits an expansion of the form

$$
\mathbf{E}^{\mathrm{ext}}(\mathbf{r}, \omega)=\sum_{L} \mathbf{E}_{L}^{\mathrm{ext}} j_{L}(k \mathbf{r}),
$$

where the coefficients 


$$
\mathbf{E}_{L}^{\mathrm{ext}}=\sum_{L^{\prime}} \mathbf{L}_{L L^{\prime}} \psi_{L^{\prime}}^{M, \mathrm{ext}}+\mathrm{i} \sum_{L^{\prime} L^{\prime \prime}} \mathbf{N}_{L L^{\prime \prime}}^{*} \times \mathbf{L}_{L^{\prime \prime} L^{\prime}}^{*} \psi_{L^{\prime}}^{E, \mathrm{ext}}
$$

are obtained from the above expressions. Similar formulas are obtained for $\mathbf{H}^{\text {ext }}$ and for the induced fields $\mathbf{E}^{\text {ind }}$ and $\mathbf{H}^{\text {ind }}$ in terms of multipole coefficients. Finally, we insert them into Eq. (2) and perform the integral over a sphere in the $s$ $\rightarrow \infty$ limit. Then, the first two terms inside the integrand give a vanishing contribution because the induced far-field is transverse. The remaining part of the integral can be recast, noticing that only real terms must be retained

$$
\begin{aligned}
\mathbf{F}(\omega)= & \frac{1}{(4 \pi k)^{2}} \sum_{L L^{\prime}} \operatorname{Re}\left\{\hat{\mathbf{n}}_{L L^{\prime}} \times\left(\mathrm{i}\left[\mathbf{E}_{L}^{\mathrm{ext}}\left(\mathbf{E}_{L^{\prime}}^{\mathrm{ind}}\right) *+\mathbf{H}_{L}^{\mathrm{ext}}\left(\mathbf{H}_{L^{\prime}}^{\mathrm{ind}}\right) *\right]\left(1-(-1)^{l}\right)-\mathrm{i}\left[\mathbf{E}_{L}^{\mathrm{ind}}\left(\mathbf{E}_{L^{\prime}}^{\mathrm{ext}}\right) *+\mathbf{H}_{L}^{\mathrm{ind}}\left(\mathbf{H}_{L^{\prime}}^{\mathrm{ext}}\right) *\right]\left(1-(-1)^{l^{\prime}}\right)\right.\right. \\
& \left.\left.+2\left[\mathbf{E}_{L}^{\text {ind }}\left(\mathbf{E}_{L^{\prime}}^{\text {ind }}\right) *+\mathbf{H}_{L}^{\text {ind }}\left(\mathbf{H}_{L^{\prime}}^{\text {ind }}\right) *\right]\right)\right\},
\end{aligned}
$$

where

$$
\hat{\mathbf{n}}_{L L^{\prime}}=\int d \Omega Y_{L^{\prime}}^{*}(\Omega) \hat{\mathbf{n}}(\Omega) Y_{L}(\Omega)
$$

and $\hat{\mathbf{n}}(\Omega)=\sqrt{4 \pi / 3}\left[(\hat{\mathbf{x}}+\mathrm{i} \hat{\mathbf{y}}) Y_{1-1} / \sqrt{2}-(\hat{\mathbf{x}}-\mathrm{i} \hat{\mathbf{y}}) Y_{11} / \sqrt{2}+\hat{\mathbf{z}} Y_{10}\right]$ is the radial vector as a function of the polar direction $\Omega$.

\section{RESULTS AND DISCUSSION}

Figure 2 shows the dependence of the momentum transfer on electron impact parameter $b$ for alumina and silver spheres of different radius, as calculated from Eqs. (1) and (9). Measured optical data have been used for the dielectric function of these materials. ${ }^{7}$ One can observe a nearly exponential decay of the momentum transfer with $b$. Besides, the momentum transferred along the direction of the electron velocity vector ( $\Delta p_{z}$, dashed curves) is generally smaller than the remaining perpendicular component $\left(\Delta p_{x}\right.$, solid curves), which finds an explanation in the fact that the contribution of these components to the energy loss $\hbar \omega$ is $v \Delta p_{z}+\left(\Delta p_{x}\right)^{2} / m$, where $m$ is the electron mass: since $m v \gg \Delta p, \Delta p_{x}$ is allowed to take larger values than $\Delta p_{z}$ for each fixed $\omega$.

Notice also that $\Delta p_{x}$ converges quickly to the large $b$ limit [Eq. (6), dotted curves], producing a finite result under the scaling of Fig. 2, unlike $\Delta p_{z}$, which goes faster to 0 for large $b$. In this limit, the electron induces a dipole in the particle directed toward the electron, which results in an attractive force between these two similar to the image potential at surfaces ${ }^{20}$ leading to a momentum transfer $\Delta \mathbf{p} \approx \Delta p_{x} \hat{\mathbf{x}}$. For small metallic particles and closer encounters this picture is no longer valid and $\Delta p_{x}$ can actually reverse its sign and have a net repulsive behavior (e.g., in Fig. 2 for Ag particles of radius $a=10 \mathrm{~nm}$ and also for the fullerenes of Fig. 4).

A more detailed analysis of the magnitude of the momentum transfer effect is given in Fig. 3. The momentum transfer is normalized to the particle mass $M$ and the result is the change in the particle velocity induced by the passage of the electron as a function of particle radius $a$. The trajectory of the $200-\mathrm{keV}$ electron under consideration passes $10 \mathrm{~nm}$ away from the surface of the spherical alumina particles. The full-multipole calculation [Eqs. (1) and (9), solid curves] agrees well with the small particle limit [Eqs. (1) and (5), dashed curves] when $a$ is much smaller than $b-a=10 \mathrm{~nm}$. Even though the electron-particle interaction increases with the radius $a$, the actual change in the particle velocity shows a nearly exponential decay with increasing $a$.

In a situation where the particle is trapped by lasers (e.g., in optical tweezers ${ }^{4}$ or in optical stretchers ${ }^{5}$ ), one should compare the interaction with the electrons to the interaction with the laser light. To this end, we will consider a trapping cw-Ti:sapphire 100-mW laser emitting at a wavelength $\lambda$ $=785 \mathrm{~nm}$ and focused on a region of radius $R_{f}=10 \mu \mathrm{m}$. Furthermore, we will contemplate the momentum transferred by the laser during the average time span $\Delta t$ between two consecutive passing electrons in a transmission electron microscope operating at a current of $1 \mathrm{nA}$. The particle polarizability $\alpha$ is all that is needed to calculate light forces for the small radii under discussion $(a \ll \lambda)$, according to Eq. (3). Now, for real $\alpha$ this equation defines a conservative gradient force that responds to the potential $-(\alpha / 2)|\mathbf{E}|^{2}$, where $\mathbf{E}$ is the laser light field, whereas the imaginary part of $\alpha$ represents photon absorption by the particle that translates into light pressure. ${ }^{21}$ These two components are represented sepa-

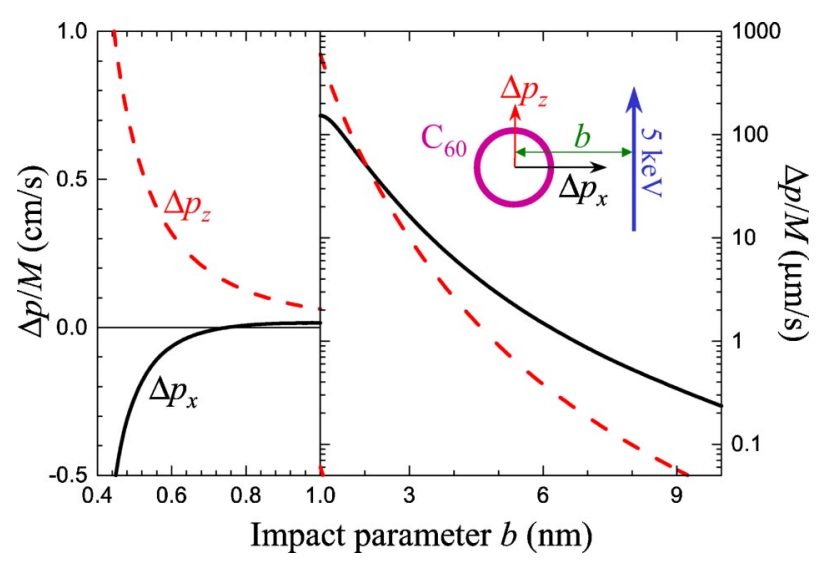

FIG. 4. (Color online). Momentum transferred from a 5-keV electron to a $\mathrm{C}_{60}$ cluster as a function of impact parameter $b$. The momentum is normalized to the cluster mass $M$. 
rately in Fig. 3 after multiplication by $\Delta t / M$ (dotted curves). The light pressure contribution is calculated for an incidence plane wave with the same photon flux as the laser at its focus. The gradient force component is obtained from the maximum force in the focus region assuming a Gaussian profile for the laser field intensity (i.e., $|\mathbf{E}|^{2} \propto \exp \left[-R^{2} /\right.$ $\left.\left.\left(R_{f} / \ln 2\right)^{2}\right]\right)$. Finally, it is convenient to define the polarizability from its relation to the scattering matrix, which upon inspection permits writing $\alpha=3 t_{1}^{E} / 2 k^{3}$. Unlike the wellknown expression ${ }^{12} \alpha=a^{3}(\epsilon-1) /(\epsilon+2)$, the former relation predicts a nonzero value for $\operatorname{Im}\{\alpha\}$ even for particles with real $\epsilon$ (like our alumina spheres), arising as a pure retardation correction associated to radiation scattering (this is actually the origin of the light pressure component of Fig. 3). (Incidentally, gravity would produce a velocity change $g \Delta t$ $=1.56 \mathrm{~nm} / \mathrm{s}$, which is well compensated for in currently available optical trapping systems.)

An important conclusion that can be extracted from Fig. 3 is that the crossover of trapping light into the main source of momentum occurs for particles of $20 \mathrm{~nm}$ in diameter when the electrons pass at a distance of $10 \mathrm{~nm}$ from the particles surface, thus allowing one to perform energy loss analysis of the transmitted electrons with significant statistics. Therefore, transmission electron microscopy can be combined with in vасио optical trapping to study particles of sizes above some tens $\mathrm{nm}$.

While the transfer of momentum by the trapping light occurs in a continuous smooth fashion, the electrons deposit all of the momentum during a small time interval $\sim a / v$ ( $\ll \Delta t=0.16 \mathrm{~ns}$ for $1 \mathrm{nA}$ electron current). However, the change in particle velocity per electron (vertical scale in Fig. 3) produces a minute particle displacement during $\Delta t$ (smaller than $1.6 \times 10^{-9} \mathrm{~nm} \ll a$ ), and therefore, the effect of the passing electrons is experienced by the particle as a nearly continuous source of momentum that is describable by an average force $\Delta \mathbf{p} / \Delta t$. Actually, Fig. 3 suggests that using more intense electron beams (with even smaller impact parameters) acting during periods of the order of one second will still not produce ejection of the particles from their trapping locations.

It should be stressed that the momentum transfers that we have calculated using classical electromagnetic theory must be understood as the average value over many incoming electrons, since the actual strength of the interaction is not large enough as to guarantee that many photons are exchanged between each electron and a given particle. Like in aloof EELS experiments, ${ }^{8}$ most electrons will not interact with the particles at all, so that the present results must be understood under the perfectly valid perspective of a statistical average performed over many beam electrons. The quadratic deviation from these average forces can also play a role (similar to straggling in stopping power theory), but this subject is left for future consideration.

We have also studied momentum transfer to $\mathrm{C}_{60}$ clusters (Fig. 4). The scattering matrices $t_{l}^{\nu}$ have been obtained within the discrete-dipole approximation, ${ }^{22,23}$ where each carbon atom is described by an induced dipole whose polarizability is fitted to reproduce correctly the measured optical response of graphite. ${ }^{7}$ Further details concerning the procedure fol-

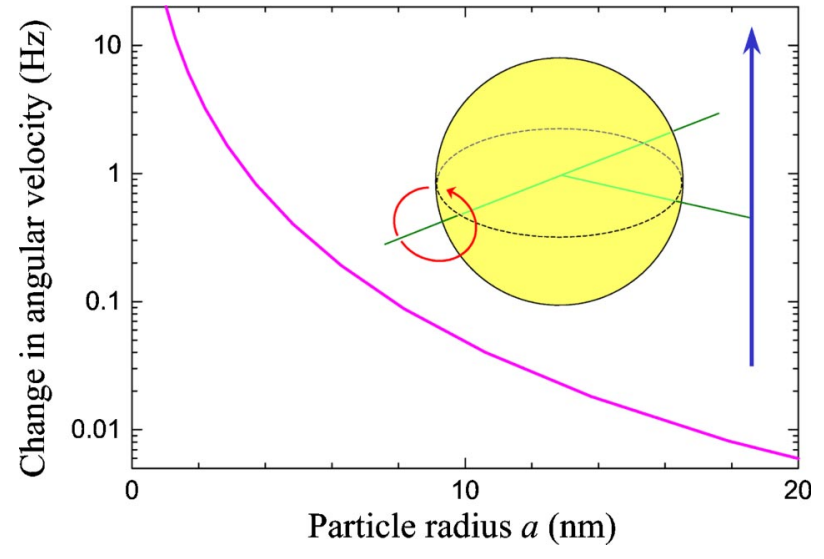

FIG. 5. (Color online). Change in the particle angular velocity as a result of the torque exerted by the electron. The particle is made of $\mathrm{Al}_{2} \mathrm{O}_{3}$ (density $\rho=4.02 \mathrm{~g} / \mathrm{cm}^{3}$ ), the electron energy is $200 \mathrm{keV}$, and the distance from the trajectory to the particle surface is $10 \mathrm{~nm}$.

lowed to obtain $t_{l}^{\nu}$ will be given elsewhere. ${ }^{24}$ At relatively small interaction distances $b$, the $z$ component of the momentum is larger than the $x$ component and the latter is negative. These are effects that can be hardly found in the above examples and that originate in high-order multipoles (actually, $l \leqslant 5$ are needed for convergence within the range of $b$ under consideration). Even at a distance of $9 \mathrm{~nm}$ (notice that $\mathrm{C}_{60}$ has a diameter of only $0.7 \mathrm{~nm}$ ) the change in velocity produced by the passing electron can be substantial. Therefore, the interaction of fast electrons with small clusters can produce dramatic effects if these are not mightily bound by a mechanism stronger than optical trapping.

Finally, the passing electron can induce a torque on the particle that changes its angular momentum $\left(\Delta L_{y}\right)$ and makes it rotate. This is the effect discussed in Fig. 5. Like the electromagnetic force above, the torque $\mathbf{G}$ is obtained from the integral of Maxwell's stress tensor, ${ }^{12}$ which yields

$$
\mathbf{G}=\frac{-s^{3}}{4 \pi} \operatorname{Re}\left\{\int_{S} d \hat{\mathbf{s}}[(\hat{\mathbf{n}} \cdot \mathbf{E}) *(\mathbf{E} \times \hat{\mathbf{n}})+(\hat{\mathbf{n}} \cdot \mathbf{H}) *(\mathbf{H} \times \hat{\mathbf{n}})]\right\},
$$

where $s$ is the radius of a sphere where the particle is embedded. Proceeding in a way similar to the derivation of the force presented in Sec. II, this expression can be written in terms of multipole components as

$$
\begin{aligned}
\mathbf{G}= & \frac{1}{4 \pi k^{3}} \sum_{l m l^{\prime} m^{\prime}} l(l+1) \operatorname{Re}\left\{\left[\frac{1}{2} \sqrt{(l+m+1)(l-m)} \delta_{m+1, m^{\prime}}(\hat{\mathbf{x}}\right.\right. \\
& \left.-\mathrm{i} \hat{\mathbf{y}})+\frac{1}{2} \sqrt{(l-m+1)(l+m)} \delta_{m-1, m^{\prime}}(\hat{\mathbf{x}}+\mathrm{i} \hat{\mathbf{y}})+m \delta_{m m^{\prime}} \hat{\mathbf{z}}\right] \\
& \times\left[\psi_{L}^{E \text {,ind }}\left(\psi_{L^{\prime}}^{E \text {,ind }}\right) *+\psi_{L}^{M, \text { ind }}\left(\psi_{L^{\prime}}^{M, \text { ind }}\right) *\right. \\
& \left.\left.+\mathrm{i} \psi_{L}^{E \text {,ind }}\left(\psi_{L^{\prime}}^{E, \text { ext }}\right) *+\mathrm{i} \psi_{L}^{M, \text { ind }}\left(\psi_{L^{\prime}}^{M, \text { ext }}\right) *\right]\right\} .
\end{aligned}
$$

Equation (11) has been used to obtain Fig. 5, which shows the change in angular velocity of the particle per incident 
electron, $\Delta \Omega=\Delta L_{y} / I$, where $I=(2 / 5) a^{2} M$ is the moment of inertia of the alumina sphere.

Averaging over the electrons of a $1 \mathrm{nA}$ electron beam passing at $10 \mathrm{~nm}$ from the surface of an alumina sphere of radius $a=20 \mathrm{~nm}$, one finds an angular acceleration of $39 \mathrm{MHz} / \mathrm{s}$. Under these conditions, the linear momentum transferred by the electrons can be absorbed by the trapping light, as discussed above. However, the angular momentum is not absorbed, and the particle will spin with increasing angular velocity until either the centrifugal force breaks it apart or radiation emission at the rotation frequency (vacuum friction) compensates for the electron-induced torque.

In conclusion, we have shown that fast electrons following aloof trajectories (i.e., without direct overlap with the sample) in a transmission electron microscope can exert time-averaged forces on small particles of similar magnitude as those forces associated to trapping in optical tweezers and stretchers, and therefore, this effect can be used for analytical studies of mechanical properties of such particles, while electron energy loss spectra can be actually taken without causing ejection of the particles from their trapping positions. The experimental challenge consists in combining electron microscopy with optical trapping in vacuum, and we hope that this work can contribute to stimulate further research in this direction. Besides, the linear and angular momentum transfers discussed here can play a relevant role in other types of trapping, like for particles deposited on a substrate, or for particles trapped by a tip, where the forces and torques induced by the passing electrons can push, pull, reposition, or reorient the trapped particles.

\section{ACKNOWLEDGMENTS}

The author wants to thank G. G. Hembree for suggesting this subject and for helpful and enjoyable discussions. This work has been partially supported by the Basque Departamento de Educación, Universidades e Investigación, the University of the Basque Country UPV/EHU (Contract No. 00206.215-13639/2001), and the Spanish Ministerio de Ciencia y Tecnología (Contract No. MAT2001-0946).
${ }^{1}$ L. Novotny, R. X. Bian, and X. S. Xie, Phys. Rev. Lett. 79, 645 (1997).

${ }^{2}$ J.-C. Meiners and S. R. Quake, Phys. Rev. Lett. 84, 5014 (2000).

${ }^{3}$ P. T. Korda, M. B. Taylor, and D. G. Grier, Phys. Rev. Lett. 89, 128301 (2002).

${ }^{4}$ See D. G. Grier, Nature (London) 424, 810 (2003) and references therein.

${ }^{5}$ J. Guck, R. Ananthakrishnan, T. J. Moon, C. C. Cunningham, and J. Käs, Phys. Rev. Lett. 84, 5451 (2000).

${ }^{6}$ P. E. Batson, N. Dellby, and O. L. Krivanek, Nature (London) 418, 617 (2002).

${ }^{7}$ E. D. Palik, Handbook of Optical Constants of Solids (Academic Press, New York, 1985);A. Howie and R. H. Milne, Ultramicroscopy 18, 427 (1985);R. H. Ritchie and A. Howie, Philos. Mag. A 58, 753 (1988);A. Howie and C. Walsh, Microsc. Microanal. Microstruct. 2, 171 (1991).

${ }^{8}$ F. J. García de Abajo, N. Zabala, A. G. Pattantyus-Abraham, A. Rivacoba, M. O. Wolf, and P. M. Echenique, Phys. Rev. Lett. 91, 143902 (2003).

${ }^{9}$ M. M. Burns, J.-M. Fournier, and J. A. Golovchenko, Phys. Rev. Lett. 63, 1233 (1989).

${ }^{10}$ M. M. Burns, J.-M. Fournier, and J. A. Golovchenko, Science 249, 749 (1990).

${ }^{11}$ M. Greiner, O. Mandel, T. Esslinger, T. W. Hänsch, and I. Bloch,
Nature (London) 415, 39 (2002).

${ }^{12}$ J. D. Jackson, Classical Electrodynamics (Wiley, New York, 1975).

${ }^{13}$ A. Rivacoba and P. M. Echenique, Ultramicroscopy 26, 389 (1988).

${ }^{14}$ See course notes by C. Cohen-Tannoudji, available at http:// www.lkb.ens.fr/ cct/

${ }^{15}$ F. J. García de Abajo, Phys. Rev. B 59, 3095 (1999).

${ }^{16}$ T. A. Nieminen, H. Rubinsztein-Dunlop, N. R. Heckenberg, and A. I. Bishop, Comput. Phys. Commun. 142, 468 (2001).

${ }^{17}$ F. J. García de Abajo, Phys. Rev. Lett. 82, 2776 (1999).

${ }^{18}$ M. Abramowitz and I. A. Stegun, Handbook of Mathematical Functions (Dover Publications, New York, 1972).

${ }^{19}$ A. Messiah, Quantum Mechanics (North-Holland, New York, 1966).

${ }^{20}$ N. Barberan, P. M. Echenique, and J. Vias, J. Phys. C 12, L111 (1979); P. M. Echenique and A. Howie, Ultramicroscopy 16, 269 (1985); F. J. García de Abajo and P. M. Echenique, Phys. Rev. B 46, 2663 (1992).

${ }^{21}$ C. N. Cohen-Tannoudji, Rev. Mod. Phys. 70, 707 (1998).

${ }^{22}$ L. Henrard and Ph. Lambin, J. Phys. B 29, 5127 (1996).

${ }^{23}$ A. Rivacoba and F. J. García de Abajo, Phys. Rev. B 67, 085414 (2003).

${ }^{24}$ F. J. García de Abajo et al. (unpublished). 